\title{
A new type Lyot filter insensitive to incident angle
}

\author{
Liping Zhang • Yong Kong • Chen Deng • \\ Yuming Wang
}

Received: 22 October 2011 / Accepted: 12 June 2012 / Published online: 3 July 2012

(C) The Author(s) 2012. This article is published with open access at Springerlink.com

\begin{abstract}
The shifting direction of a central wavelength for a quartz birefringent filter is found different when the filter is rotated around the axis parallel and perpendicular to the quartz optical axis in this paper. Based on this result, a new type of two-stage modified-Lyot quartz filter is presented, which show that the central wavelength of this new type Lyot filter is actually insensitive to incident angle through theory analysis when incident angle is less than $18^{\circ}$, and the maximum transmission decrease only about $66 \%$ compared to normal incidence. Moreover, this conclusion is in good agreements with relative experiment results.
\end{abstract}

Keywords Incident angle $\cdot$ Quartz plate $\cdot$ Birefringent $\cdot$ Filter

\section{Introduction}

There are two classic types of optical birefringence filters: Lyot filter (Evans 1949; Aharon and Abdulhalim 2009) and Solc filter (Evans 1958; Solc 1965). Compared to other optical filters, the unique advantage offered by this birefringence filter is the tenability to a desired wavelength, low insertion loss and wide field of view. Thus, birefringence filters have been

This work was supported by the funding program of Shanghai Education Commission with the project number 10YZ171 and also supported by the subject and specialty construction of broadcast television communication web of Shanghai Education Commission funding program in China.

L. Zhang $\cdot$ Y. Kong $(\varangle) \cdot$ C. Deng $\cdot$ Y. Wang

College of Electrical and Electronic Engineering, Shanghai University of Engineering Science,

Long Teng road 333\#, Shanghai 201620, China

e-mail: kkyy7757@yahoo.com.cn

L. Zhang

e-mail:ky7757@sohu.com

C. Deng

e-mail: shgjddc2010@yahoo.cn

Y. Wang

e-mail: evafyy@hotmail.com 
widely used in the fields of spectral images (Tsumura et al. 1999), laser tuning (Wang and Yao 1992; Wacogne et al. 1993), remote sensing (Green et al. 1995; Rock et al. 1986), astronomy (Hobbs 1993; Miller 19990; Kopp et al. 1997), dense wavelength division multiplexing (Juan et al. 2004; Luo and Wan 2011) and so on.

Working on a fixed transmission wavelength is important for birefringence filters. For example, in solar observation instruments, transmission function distortion under off-axis incidence is unacceptable (Ma et al. 2004; Fineschi and Capobianco 2011), the biologic cell's component information acquired by birefringent filter must be certain, which is can not changed with incident angle of birefringent filter (Gebhart and Mahadevan-Jansen 2006). The transmission character of birefringence filters with a titled angle has widely been studied in many papers (Lien 1990; Zhu 1994; Zhang 2000; Zhou et al. 2003), but there are few reports about how to maintain a fixed transmission central wavelength when the angle of incident light is changed. In this paper, a detailed research on this subject is reported. It is shown that the central wavelength of our designed new-type filter is actually insensitive to incident angle according to the relative theory and experiment results.

\section{Numerical simulation and theory analysis}

The components shown in Fig. 1 consist of Glan prism $P_{1}, P_{2}$ and the quartz plate $L_{1}$. The azimuth angle between the polarization plane of $P_{1}$ and the optical axis of the quartz plate or $Z$ axis is $45^{\circ}$, which is the same as $P_{2}$. It is obvious that the optical axis of the quartz plate is parallel to the $Z$ axis here. After setup 1 of Fig. 1 is rotated around the axis vertical to the quartz optical axis with angle $i$, then it become Fig. 2 as setup 2. In this case the description formula for birefringence of the quartz plate and intensity of the output light in setup 2 (Zhu 1994; Zhang 2000) is:

$$
\begin{gathered}
\delta_{1}=\frac{2 \pi d}{\lambda}\left[\left(\sqrt{\left.\left.\frac{\left(n_{o}^{2}-n_{e}^{2}\right)^{2} \sin ^{2} i}{n_{o}^{4}}+\frac{n_{o}^{2} n_{e}^{2}}{n_{o}^{2}-\sin ^{2} i}\right)-\frac{n_{o}^{2}}{\sqrt{n_{o}^{2}-\sin ^{2} i}}-\frac{\left(n_{e}-n_{o}\right) \sin ^{2} i}{n_{o} \sqrt{n_{o}^{2}-\sin ^{2} i}}\right]}\right.\right. \\
T=\cos ^{2}\left(\frac{\delta_{1}}{2}\right)
\end{gathered}
$$

Fig. 1 Construction diagram of a single-stage Lyot filter with an optical axis of a quartz plate parallel to the $Z$ axis

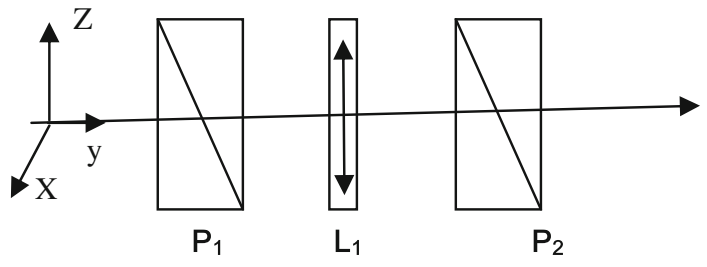

Fig. 2 Construction diagram of Fig. 1 rotated around the axis vertical to the quartz optical axis with angle $i$

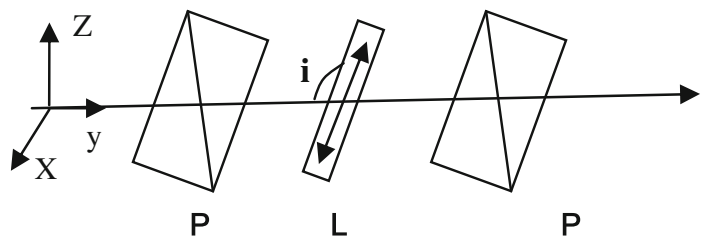


Fig. 3 Construction diagram of a single-stage Lyot filter with an optical axis of a quartz plate parallel to the $X$ axis

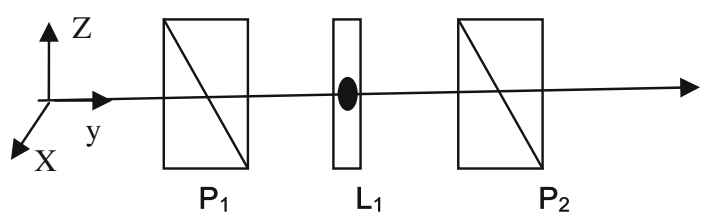

Fig. 4 Construction diagram of Fig. 3 rotated around the axis parallel to the quartz optical axis with angle $i$

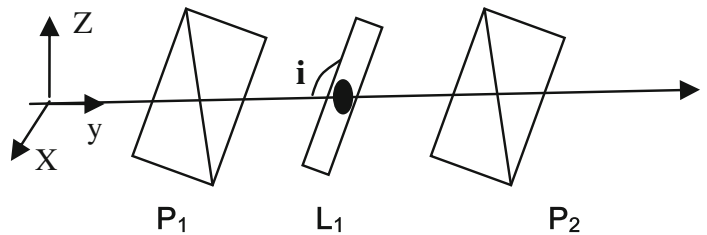

Fig. 5 Transmission spectrum of setup 1-4

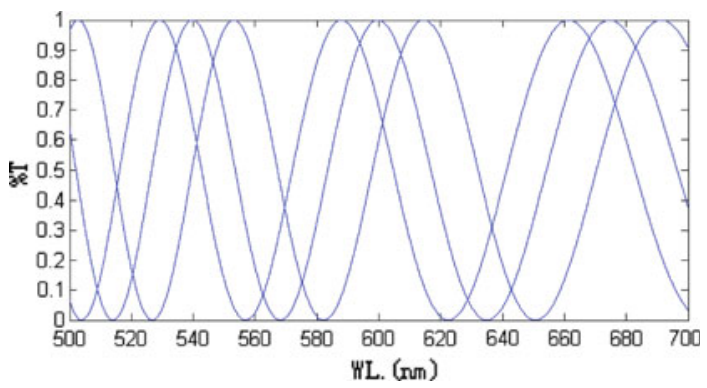

where $\lambda$ is the wavelength of incident light in vacuum, $n_{e}$ and $n_{o}$ are the extraordinary and ordinary refraction for the quartz plate respectively, and $\mathbf{d}$ is the quartz plate thickness.

The azimuth angle between the polarization plane of $P_{1}$ and optical axis of the quartz plate or $Z$ is $135^{\circ}$ in Fig. 3, which is the same as $P_{2}$. And the optical axis of the quartz plate is parallel to the $X$ axis here. After setup 3 of Fig. 3 is rotated around the axis parallel to the quartz optical axis with angle $i$, then it becomes Fig. 4 as setup 4 (Fig. 5). In this case the description formula for birefringence of the quartz plate and intensity of the output light in setup 4 (Zhu 1994; Zhang 2000) is:

$$
\begin{gathered}
\delta_{2}=\frac{2 \pi d}{\lambda}\left[\sqrt{n_{e}^{2}-\sin ^{2} i}-\sqrt{n_{o}^{2}-\sin ^{2} i}\right] \\
T=\cos ^{2}\left(\frac{\delta_{2}}{2}\right)
\end{gathered}
$$

The parameter of the quartz plate used is as follows: $n_{e}, n_{o}$ and $\mathrm{d}$ are $1.57,1.561,0.6 \mathrm{~mm}$ respectively. One of the three curves with a center wavelength at $600 \mathrm{~nm}$ is the transmission spectrum of setup 1 or setup 3, in other words, if setup 1 or setup 3 are not rotated, whether to setup 1 or to setup 3 , the transmission spectrum will be the same one. The curve with a center wavelength at about 610 and $590 \mathrm{~nm}$ is the transmission spectrum of setup 2 and setup 4 respectively for $i=18^{\circ}$. That is, compared to setup 1 and setup 2 , the center wavelength will shift to shorter or longer wavelengths corresponding to setup 3 and setup 4 respectively. So a new-type filter is presented, showed in Fig. 6 through combining setup 2 and setup 4, which will make the center wavelength not sensitive to the incident angle as we assume. 
Fig. 6 Construction diagram of a two-stage Lyot filter combining with setup 2 and setup 4

Fig. 7 Transmission spectrum of setup 6 for $i=0^{\circ}$ and $i=18^{\circ}$
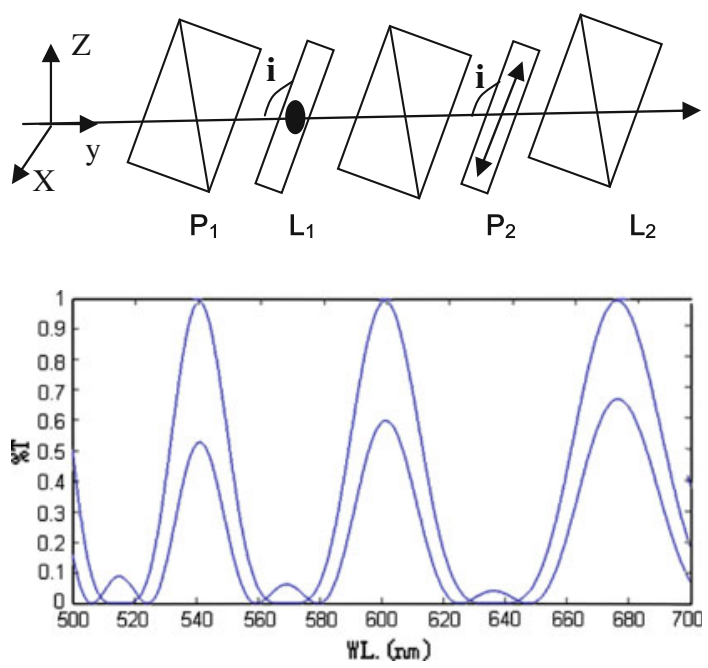

Transmissivity of output light of setup 6 of Fig. 6 is:

$$
T=\cos ^{2}\left(\frac{\delta_{1}}{2}\right) \cos ^{2}\left(\frac{\delta_{2}}{2}\right)
$$

The spectrum with higher and lower transmissivity illustrated in Fig. 7 are the simulation results of output light intensity of setup 6 for $i=0^{\circ}$ and $i=18^{\circ}$. We can find that the central wavelength of this new type Lyot filter is actually insensitive to incident angle through theory analysis when incident angle is more than $18^{\circ}$, and the maximum transmission decrease only about $66 \%$ compared to normal incidence, and the tradeoff in maximum transmission can be accepted by some application as reference (Ma et al. 2004; Fineschi and Capobianco 2011; Gebhart and Mahadevan-Jansen 2006). Which demonstrates this type filter of setup 6 is actually not sensitive to incident angle.

\section{Experimental results and discussion}

The parameter of quartz used is same as the above value. The spectral photometer we used is domestic, its type is $\mathrm{V}-1600 \mathrm{PC}$ with $1 \mathrm{~nm}$ sweeping step and $300-1,100 \mathrm{~nm}$ sweeping range. From Figs. 8 and 9, we can find the results of experiment are very similar to the results of Figs. 5 and 7 obtained by numerical simulation, which verify the truth of our assumption. But for Fig. 10, we can find the central wavelength shift to other values when the incident angle changes to $45^{\circ}$ (It is deserved to be mentioned that the critical semi-field angle of Glan prism is about $20^{\circ}$ (Zhu 1994), and here we do not consider the effect brought by titled Glan prism, but only consider the $45^{\circ}$ rotation of quartz plate), which is because the shift value of central wavelength with an increasing incident angle is so much that the product between one order of central wavelength of setup 2 and the next order of central wavelength of setup 4 is a more prominent influence factor than the product between the one order of central wavelength of setup 2 and the corresponding order of central wavelength of setup 4. To explain clearly, we can find there have some sideband spectrums illustrated in Figs. 7 and 9; it will become larger when the rotated angle of quartz increases, which is caused by the reason of above 
Fig. 8 Experiment result of transmission spectrum of setup $1-4$

Fig. 9 Experiment result of transmission spectrum of setup 6 for $i=0^{\circ}$ and $i=18^{\circ}$
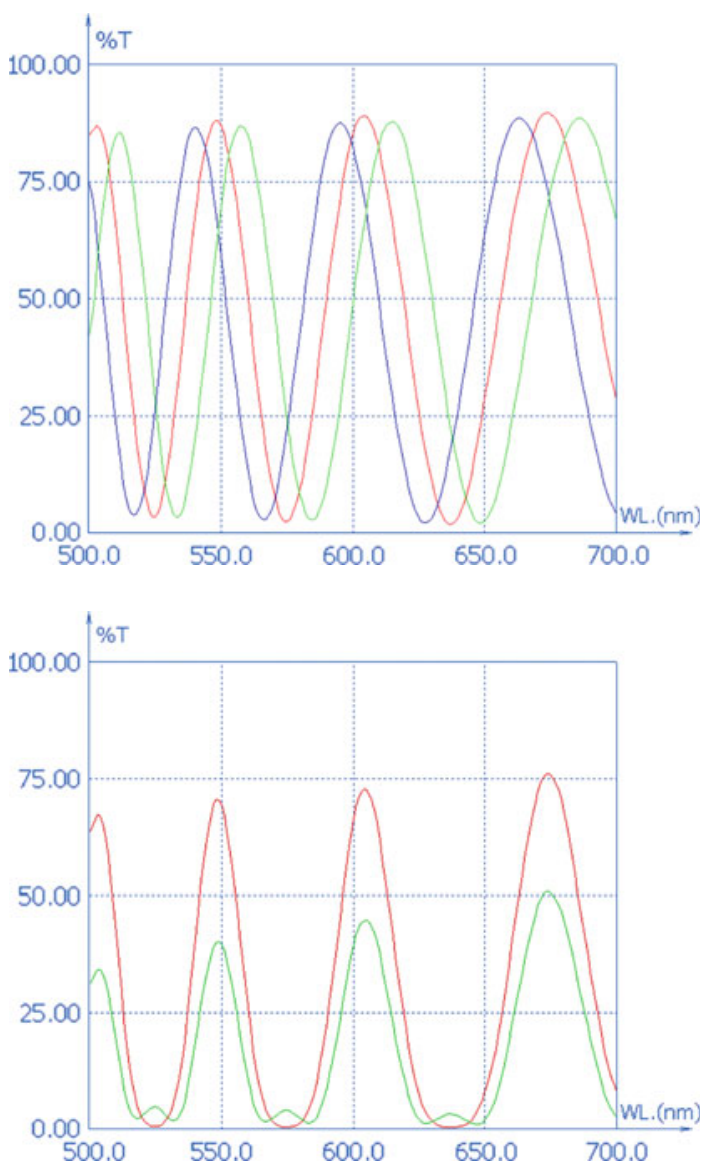

Fig. 10 Experiment result of transmission spectrum of setup 6 for $i=45^{\circ}$ but only the quartz plate is rotated and the Glan prism is not rotated

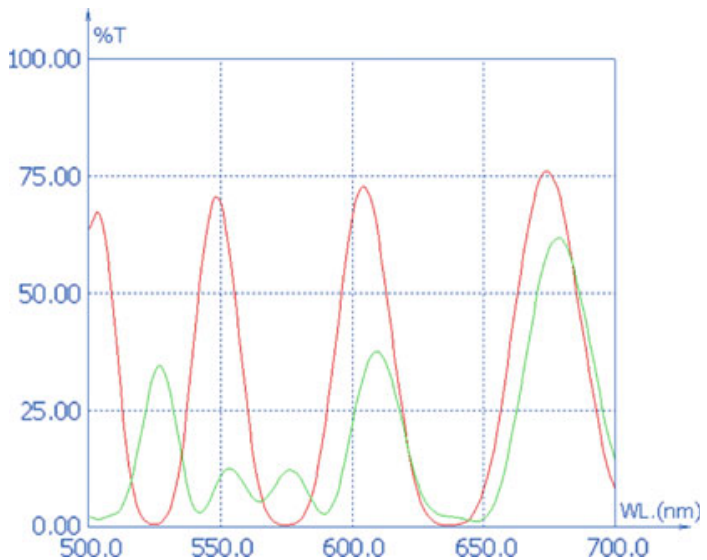

analysis. So it is important to hold the incident angle less than about $72^{\circ}$ in case the central wavelength will change to too large.

In another situation as Figs. 11 and 12, because the transmission formula of Fig. 11 is the same to Fig. 4 and the transmission formula of Fig. 12 is the same to Fig. 2 respectively 
Fig. 11 Construction diagram of Fig. 1 rotated around the axis parallel to the quartz optical axis with angle $i$
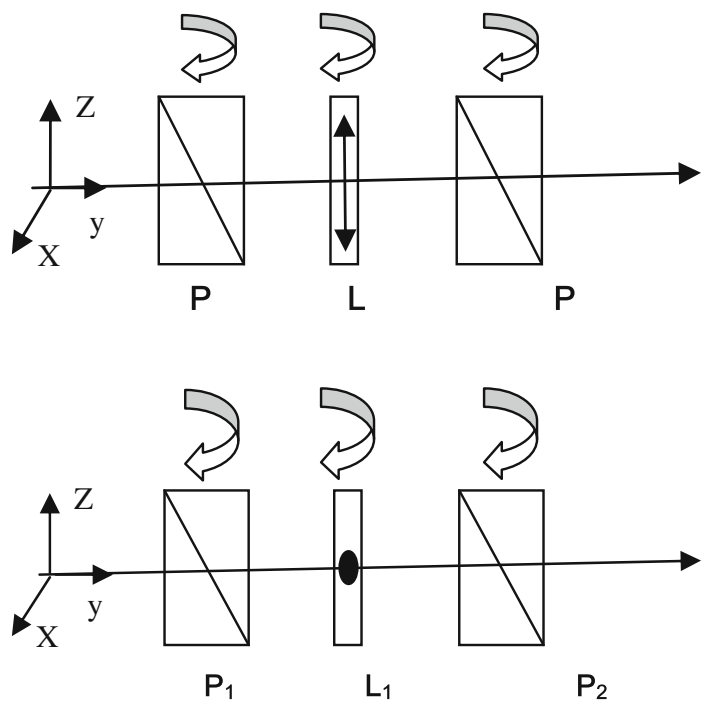

Fig. 12 Construction diagram of Fig. 3 rotated around the axis vertical to the quartz optical axis with angle $i$

Fig. 13 Construction diagram of a two-stage Lyot filter combining with Figs. 11 and 12

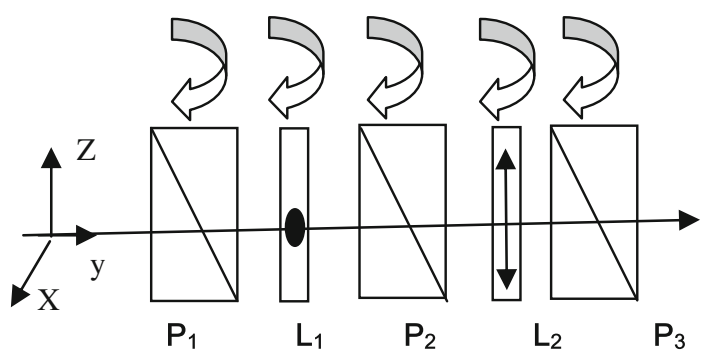

(Zhu 1994; Zhang 2000). The transmission spectrum of Fig. 13 as setup 13 will be the same to Fig. 6, which is also verified by our analysis of theory and experiment. In conclusion, whether to the construction of setup 13 or setup 6, the central wavelength is insensitive to the incident angle.

\section{Conclusion}

A new type of two-stage modified-Lyot filter is presented, which is composed of two quartzes with a vertical optical axis to each other and three Glan prisms in sequence as Figs. 6 and 13. The azimuth angle of all polarization planes of three Glan prisms is parallel, and the azimuth angle of all polarization planes of three Glan prisms to the optical axis of the first and the second quartz plate are $45^{\circ}$ and $135^{\circ}$ respectively. It shows that the central wavelength of this new-type filter is insensitive to incident angle whether the equipment rotated around the axis is parallel or vertical to the optical axis of the quartz plate according to theory and experiment results. These results will have an extensive application to design the relative birefringent filter with insensitive incident angles in fields such as spectral images, laser tuning, astronomy and so on. 
Open Access This article is distributed under the terms of the Creative Commons Attribution License which permits any use, distribution, and reproduction in any medium, provided the original author(s) and the source are credited.

\section{References}

Aharon, O., Abdulhalim, I.: Liquid crystal Lyot tunable filter with extended free spectral range. Opt. Express 17, 11426-11433 (2009)

Evans, J.W.: The birefringent filter. J. Opt. Soc. Am. 39, 229-242 (1949)

Evans, J.W.: Solc birefringent filter. J. Opt. Soc. Am. 48, 142-145 (1958)

Fineschi, S., Capobianco, G.: Liquid crystals Lyot filter for solar coronagraphy. SPIE 8148, 814808.1814808.10 (2011)

Gebhart, S.C., Mahadevan-Jansen, A.: Brain tumor demarcation with liquid-crystal tunable filter spectral imaging. SPIE 6080, 608001.1-608001.12 (2006)

Green, R., Sarture, C., Chovit, C., Faust, J., Hajek, P., Novak, H.: AVIRIS: a new approach to earth remote sensing. Opt. Photon. News 6, 30-33 (1995)

Hobbs, J.: Tunable liquid-crystal filter helps study stellar emission. Laser Focus World 29, 29-30 (1993)

Kopp, G.A., Derks, M.J., Elmore, D.F., Hassler, D.M., Woods, J.C., Streete, J.L., Blankner, J.G.: Tunable liquid-crystal filter for solar imaging at the He i 1083-nm line. Appl. Opt 36, 291-296 (1997)

Lien, A.: Extended Jones matrix representation for the twisted nematic liquid-crystal display at oblique incidence. Appl. Phys. Lett. V57(N26), 2767-2768 (1990)

Luo, Z., Wan, Z.: Design and tolerance analysis of optical interleaver based on retardant crystals. Optik 122, 133-135 (2011)

Ma, J., Wang, J., Cao, W., Denker, C., Wang, H.: Near infrared (NIR) achromatic phase retarder. SPIE 523, 193247 (2004)

Miller, P.: Tunable narrowband birefringent filters for astronomical imaging. In: Crawford, D.L. (ed.) Instrumentation in Astronomy VII, Proceedings of SPIE, vol. 1235, pp. 466-473 (1990)

Rock, B.N., Vogelmann, J.E., Williams, D.L., Vogelmann, A.F., Hoshizaki, T.: Remote detection of forest damage. Bioscience 36, 439-445 (1986)

Solc, I.: Birefringent chain filters. J. Opt. Soc. Am. 55, 621-625 (1965)

Tsumura, N., Sato, H., Hasegawa, T., Haneishi, H., Miyake, Y.: Limitation of color samples for spectral estimation from sensor responses in fine art painting. Opt. Rev. 1, 57-60 (1999)

Wacogne, B., Goedgebuer, J., Onokhov, A., Tomilin, M.: Wavelength tuning of a semiconductor laser using nematic liquid crystals. IEEE J. Quantum Electron. 29, 1015-1017 (1993)

Wang, X., Yao, J.: Transmitted and tuning characteristics of birefringent filters. Appl. Opt. 32, 45054508 (1992)

Zhang, W.: New phase shift formulas and stability of waveplate in oblique incident beam. Opt. Commun. 176, 9-15 (2000)

Zhang, J., Liu, L., Zhou, Y.: Optimum design of a novel electro-optically tunable birefringent interleaver filter. J. Opt. A Pure Appl. Opt. 6, 1052-1057 (2004)

Zhou, Y., Liu, L., Zhang, J., Liu, D., Luan, Z.: Nearly-off-axis transmissivity of Solc birefringent filters. J. Opt. Soc. Am. A 20(4), 733-740 (2003)

Zhu, X.: Explicit Jones transformation matrix for a tilted birefringent plate with its optical axis parallel to the plate surface. J. Appl. Opt. 33(16), 3502-3505 (1994) 